\title{
Genetic Diversity of Amaranthus dubius Mart. (Khedha) Used Tribal Place in Chhattisgarh, India
}

\author{
Deepika Chandravanshi ${ }^{1 *}$, P. K. Sharma ${ }^{2}$, C. Banjare ${ }^{3}$ and Deepa Chandravanshi ${ }^{4}$
} ${ }^{1,3}$ Dept. of Horticulture, ${ }^{2}$ Dept. of Vegetable Science and ${ }^{4}$ Dept. of Soil Water Engineering College of Agriculture, Indira Gandhi
Agriculture University, Raipur, Chhattisgarh (492 012), India

\section{Corresponding Author}

Deepika Chandravanshi

e-mail:dp.chandra.888@gmail.com

\author{
Article History \\ Article ID: AR1865e \\ Received in $16^{\text {th }}$ March, 2018 \\ Received in revised form $31^{\text {st }}$ October, 2018 \\ Accepted in final form $31^{\text {st }}$ October, 2018
}

\begin{abstract}
In India, the leaves of a large number of wild and cultivated plants are used as vegetables. They have a very high protective food value and are very easy to grow. In Chhattisgarh, the life and economy of the tribal and local people are intimately connected with the natural vegetation. The present investigation research was conduct during Rabi Season during year 2014-15 and 2015-16 at Research and Instructional Farm, Department of Horticulture, Indira Gandhi Krishi Vishwavidyalaya, Raipur (C.G.). The experiment was comprised of twenty five genotypes and check variety CO-1 of Amaranthus dubius Mart. (Khedha) laid out in Randomized Block Design (RBD) with three replications. The information was based on an ethno-botanical field study of the different parts of Chhattisgarh. Morphological characters recorded in all the genotypes as per the minimal descriptors for leafy vegetables presented in table no. 1 . The plant height varies from $5 \mathrm{~m}$ to $10 \mathrm{~m}$ among various species. Leaves are oblong to elliptical with color ranged from light to dark green with some expressing red pigment throughout the genus. The inflorescence is very prominent, colorful, terminal and contain one male flower per glomerule. The obtained data expressed maximum fibre content in variety of CO-1 (15.67\%) followed by genotype IGKB-2014-51 (15.50\%) and IGKB-2014-53 (15.13\%) was observed. Variety CO-1 and genotype IGKB-2014-51 are statically at par with each other.
\end{abstract}

Keywords: Identification, khedha, Raipur

\section{Introduction}

Khedha (Amaranrhus dubius Mart.) are leafy vegetable locally known as khedha bhaji (jari) belong to the family Amranthaceae. The green Amaranthus consist of approximately 60 species out of which about 18 species are occurring in India. There are three major producing Amaranthus species, A. caudatus, $A$. cruentus and $A$. hypochondriacus, all believed to originate from Central and South America; and three major leafy vegetable species, $A$. tricolor, $A$. dubius and $A$. blitum ( $A$. lividus), of which $A$. tricolor is thought to originate from India or Southern (Yadav et al., 2014). The plant height varies from $5 \mathrm{~m}$ to $10 \mathrm{~m}$ among various species. Leaves are oblong to elliptical with color ranged from light to dark green with some expressing red pigment throughout the genus. The inflorescence is very prominent, colorful, terminal and contain one male flower per glomerule. India about 800 species are consumed as wild edible plant saver the country (Singh and Arora, 1978). India is the largest producer of vegetable crops next to China. Leafy vegetables are cultivated in an area of 9205 thousand hectare with an annual production of 162187 thousand MT (Anon., 2013). In Chhattisgarh, vegetables occupied an area of 377.21 thousand hectare with an average production of 4965.33 thousand MT out of these, leafy vegetables are cultivated in an area of 7688 hectare with an average production of 72902 MT (Anon., 2014). Despite such a huge production in the country, less than the appropriate requirement of balanced diet is provided to every individual.

In Chhattisgarh, the life and economy of the tribal and local people are intimately connected with the natural vegetation. Leafy vegetables play a major role in the nutritional requirement of the tribal and local population in remote parts of the Chhattisgarh. The use of leafy vegetables as food has been formed an integral part of the culture and tradition of many indigenous communities of the world. It constitutes an essential component in the diet and food security of many tribal and local communities particularly people living around the forest fringe. Looking to the various types of leafy vegetables grown in Chhattisgarh i.e. Amari Bhaji, Tinpania Bhaji, Bathua Bhaji, Chaulai Bhaji, Chech Bhaji, Chunchunia Bhaji, Karmota Bhaji, Lal Bhaji, Methi Bhaji, Palak, Patawa Bhaji, Patharri Bhaji, Poi Bhaji, Sarson Bhaji, Jadi Bhaji, Bohar Bhaji and rapid urbanization of developing country like India, food security is a major concern (Chauhan et al., 2014). In Chhattisgarh state, leafy vegetables are found naturally in 
both cultivated and non-cultivated lands and there are major dietary component of tribal as well as rural people of the state. It's provides rural households with supplemental income opportunities through their sale in the markets. Vegetable amaranth serves as an alternative source of nutrition for people in developing countries since it is a rich and inexpensive source of carotenoid, protein, vitamins and dietary fibre (Shukla et al., 2003).

\section{Materials and Methods}

The field study was carried out in the villages and forest villages of the different region of Chhattisgarh. Methodology covers two types of survey namely: Field Survey and Literature Collection. The main aim of the survey was to collect information about the leafy vegetables plant species which are used by the tribal and local peoples and also the species are identified and documented by collecting samples of plant species. The total respondents were 30-35 in numbers per villages to collect information on Amaranthus dubius Mart. (Khedha Bhaji), along with utilization of wild edible plants of the study area. Raipur district is situated in the central part of Chhattisgarh, agro-climatologically known as Chhattisgarh plains and lies between $21^{\circ} 16^{\prime} \mathrm{N}$ latitude and $81^{\circ} 36^{\prime} \mathrm{E}$ longitude with an altitude of $\mathbf{2 8 9 . 5 6}$ meters above the mean sea level. Raipur, the place of investigation, comes under dry, sub-humid region. It comes under seventh agro-climatic zone of the country, i.e. eastern plateau and hills. The annual average rainfall is $1200-1400 \mathrm{~mm}$, out of which about $85 \%$ is received from third week of June to mid-September and very little during October to February. May is the hottest (46 ${ }^{\circ} \mathrm{C}$ ) and December is the coolest $\left(6^{\circ} \mathrm{C}\right)$ month of the year. The experimental site was located at Research and Instructional Farm, Department of Horticulture, College of Agriculture, Indira Gandhi Krishi Vishwavidyalaya, Raipur (C.G.) having with adequate facilities for irrigation and drainage are available.

\section{Results and Discussion}

The present investigation was undertaken to study the mean performance of Amaranthus dubius Mart. (khedha) genotypes and to assess the genetic diversity in Amaranthus dubius Mart. (khedha) genotypes for yield and its component characters (Table 1 and Figure 1). All the genotypes of khedha were evaluate for the branching index were categorized into two group viz., nine genotypes had only at base (IGKB-2014-31, IGKB-2014-34, IGKB-2014-39, IGKB-2014-42, IGKB-2014-45, IGKB-2014-49, IGKB-2014-52, IGKB-2014-55 and variety CO-1), seventeen genotypes had branches all over the stem (IGKB-2014-32, IGKB-2014-33, IGKB-2014-35, IGKB-2014-36, IGKB-2014-37, IGKB-2014-38, IGKB-2014-40, IGKB-2014-41, IGKB-2014-43, IGKB-2014-44, IGKB-2014-46, IGKB-2014-47, IGKB-2014-48, IGKB-2014-50, IGKB-2014-51, IGKB-2014-53 and IGKB-2014-54).

The stem pubescence were categorized into three group viz., one genotype had none stem pubescence (IGKB-2014-31), sixteen genotypes had low stem pubescence (IGKB-2014-32, IGKB-2014-33, IGKB-2014-36, IGKB-2014-37, IGKB-2014-38, IGKB-2014-39, IGKB-2014-40, IGKB-2014-41, IGKB-2014-44, IGKB-2014-45, IGKB-2014-46, IGKB-2014-47, IGKB-201448, IGKB-2014-49, IGKB-2014-50 and IGKB-2014-53), seven genotypes had medium stem pubescence (IGKB-2014-34, IGKB-2014-35, IGKB-2014-42, IGKB-2014-43, IGKB-201451, IGKB-2014-52 and variety CO-1), two genotypes had conspicious stem pubescence (IGKB-2014-54 and IGKB-201455)

The stem pigmentation were categorized into two group viz., twenty-three genotype had green (IGKB-2014-31, IGKB-2014-32, IGKB-2014-33, IGKB-2014-36, IGKB-2014-38, IGKB-2014-39, IGKB-2014-40, IGKB-2014-41, IGKB-2014-42, IGKB-2014-43, IGKB-2014-44, IGKB-2014-45, IGKB-2014-46, IGKB-2014-47, IGKB-2014-48, IGKB-2014-49, IGKB-2014-50, IGKB-2014-51, IGKB-2014-52, IGKB-2014-53, IGKB-2014-54, IGKB-2014-55 and variety CO-1) while, three genotypes had green with base pink (IGKB-2014-34, IGKB-2014-35, IGKB2014-37).

Leaf shape were categorized into three group viz., seven had obovate (IGKB-2014-31, IGKB-2014-42, IGKB-2014-43, IGKB-2014-45, IGKB-2014-46, IGKB-2014-47 and IGKB-201448), fourteen had cuneate (IGKB-2014-32, IGKB-2014-33, IGKB-2014-34, IGKB-2014-35, IGKB-2014-36, IGKB-2014-39, IGKB-2014-40, IGKB-2014-41, IGKB-2014-44, IGKB-2014-51, IGKB-2014-52, IGKB-2014-53, IGKB-2014-54 and IGKB-201455), five had lanceolate (IGKB-2014-37, IGKB-2014-37, IGKB2014-49, IGKB-2014-50 and variety CO-1).

The leaf pubescence were categorized into three group viz., eighteen genotypes had low leaf pubescence (IGKB-2014-31, IGKB-2014-32, IGKB-2014-33, IGKB-2014-36, IGKB-2014-37, IGKB-2014-38, IGKB-2014-40, IGKB-2014-41, IGKB-2014-44, IGKB-2014-45, IGKB-2014-46, IGKB-2014-47, IGKB-2014-48, IGKB-2014-49, IGKB-2014-50, IGKB-2014-53 and variety CO-1), six genotypes had medium leaf pubescence (IGKB-2014-34, IGKB-2014-35, IGKB-2014-42, IGKB-2014-43, IGKB-2014-51 and IGKB-2014-52), two genotypes had conspicious leaf pubescence (IGKB-2014-54 and IGKB-2014-55).

The leaf pigmentation were categorized into three group viz., eleven genotype had normal green (IGKB-2014-31, IGKB-201432, IGKB-2014-36, IGKB-2014-38, IGKB-2014-40, IGKB-201444, IGKB-2014-45, IGKB-2014-46, IGKB-2014-52, IGKB-201453 and IGKB-2014-55) while, ten genotypes had dark green (IGKB-2014-33, IGKB-2014-34, IGKB-2014-37, IGKB-2014-41, IGKB-2014-42, IGKB-2014-43, IGKB-2014-47, IGKB-2014-50, IGKB-2014-54 and variety CO-1), five genotypes had dark green with margin pigment (IGKB-2014-35, 39, IGKB-2014-48, IGKB-2014-49 and IGKB-2014-51) respectively.

The leaf colour intensity were categorized into three group viz., twelve genotype had light colour (IGKB-2014-34, IGKB2014-37, IGKB-2014-39, IGKB-2014-40, IGKB-2014-41, IGKB-2014-42, IGKB-2014-44, IGKB-2014-50, IGKB-2014-51, 


\begin{tabular}{|c|c|c|c|c|c|c|c|c|}
\hline Characters & $\begin{array}{l}\text { Collection } \\
\text { place (Village, } \\
\text { District) }\end{array}$ & $\begin{array}{l}\text { Branching in- } \\
\text { dex }\end{array}$ & $\begin{array}{l}\text { Stem } \\
\text { pubes- } \\
\text { cence }\end{array}$ & $\begin{array}{l}\text { Stem pig- } \\
\text { mentation }\end{array}$ & $\begin{array}{l}\text { Leaf } \\
\text { shape }\end{array}$ & $\begin{array}{l}\text { Leaf } \\
\text { pubes- } \\
\text { cence }\end{array}$ & Leaf pigmentation & $\begin{array}{c}\text { Fibre } \\
\text { con- } \\
\text { tent } \\
\% \\
\end{array}$ \\
\hline IGKB-2014-31 & $\begin{array}{l}\text { Basantpali, } \\
\text { Raigarh }\end{array}$ & Only at base & None & Green & Obovate & Low & Normal Green & 6.77 \\
\hline IGKB-2014-32 & $\begin{array}{l}\text { Gharghoda, } \\
\text { Raigarh }\end{array}$ & $\begin{array}{l}\text { Branches all } \\
\text { over the stem }\end{array}$ & Low & Green & Cuneate & Low & Normal Green & 4.73 \\
\hline IGKB-2014-33 & Gaitra. Tilda & $\begin{array}{l}\text { Branches all } \\
\text { over the stem }\end{array}$ & Low & Green & Cuneate & Low & Drak Green & 4.60 \\
\hline IGKB-2014-34 & $\begin{array}{l}\text { Timarlaga, } \\
\text { Raigarh }\end{array}$ & Only at base & Medium & $\begin{array}{l}\text { Green with } \\
\text { base pink }\end{array}$ & Cuneate & Medium & Drak Green & 5.47 \\
\hline IGKB-2014-35 & $\begin{array}{l}\text { Sakti, } \\
\text { Raigarh }\end{array}$ & $\begin{array}{l}\text { Branches all } \\
\text { over the stem }\end{array}$ & Medium & $\begin{array}{l}\text { Green with } \\
\text { base pink }\end{array}$ & Cuneate & Medium & $\begin{array}{l}\text { Drak green with } \\
\text { margin pigmented }\end{array}$ & 3.75 \\
\hline IGKB-2014-36 & $\begin{array}{l}\mathrm{M} \text { a } \mathrm{h} \text { a } \mathrm{s} \text { a - } \\
\text { mund, Arang }\end{array}$ & $\begin{array}{l}\text { Branches all } \\
\text { over the stem }\end{array}$ & Low & Green & Cuneate & Low & Normal Green & 7.00 \\
\hline IGKB-2014-37 & Tulsi, Tilda & $\begin{array}{l}\text { Branches all } \\
\text { over the stem }\end{array}$ & Low & $\begin{array}{l}\text { Green with } \\
\text { base pink }\end{array}$ & $\begin{array}{l}\text { Lanceo- } \\
\text { late }\end{array}$ & Low & Drak Green & 12.17 \\
\hline IGKB-2014-38 & $\begin{array}{l}\text { Tila, Abhan- } \\
\text { pur }\end{array}$ & $\begin{array}{l}\text { Branches all } \\
\text { over the stem }\end{array}$ & Low & Green & $\begin{array}{l}\text { Lanceo- } \\
\text { late }\end{array}$ & Low & Normal Green & 12.93 \\
\hline IGKB-2014-39 & $\begin{array}{l}\text { Khilora, } \\
\text { Abhanpur }\end{array}$ & Only at base & Low & Green & Cuneate & Low & $\begin{array}{l}\text { Drak green with } \\
\text { margin pigmented }\end{array}$ & 11.93 \\
\hline IGKB-2014-40 & $\begin{array}{l}\text { Sonpairi, } \\
\text { Abhanpur }\end{array}$ & $\begin{array}{l}\text { Branches all } \\
\text { over the stem }\end{array}$ & Low & Green & Cuneate & Low & Normal Green & 7.13 \\
\hline IGKB-2014-41 & $\begin{array}{l}\text { Tila, } \\
\text { Abhanpur }\end{array}$ & $\begin{array}{l}\text { Branches all } \\
\text { over the stem }\end{array}$ & Low & Green & Cuneate & Low & Dark Green & 6.87 \\
\hline IGKB-2014-42 & Gaitra. Tilda & Only at base & Medium & Purple & Obovate & Medium & Dark Green & 13.33 \\
\hline IGKB-2014-43 & $\begin{array}{l}\text { Khilora, } \\
\text { Abhanpur }\end{array}$ & $\begin{array}{l}\text { Branches all } \\
\text { over the stem }\end{array}$ & Medium & Green & Obovate & Medium & Dark Green & 13.17 \\
\hline IGKB-2014-44 & $\begin{array}{l}\text { Sonpairi, } \\
\text { Abhanpur }\end{array}$ & $\begin{array}{l}\text { Branches all } \\
\text { over the stem }\end{array}$ & Low & Green & Cuneate & Low & Normal Green & 11.70 \\
\hline IGKB-2014-45 & Tulsi, Tilda & Only at base & Low & Green & Obovate & Low & Normal Green & 9.83 \\
\hline IGKB-2014-46 & Gaitra, Tilda & $\begin{array}{l}\text { Branches all } \\
\text { over the stem }\end{array}$ & Low & Green & Obovate & Low & Normal Green & 10.80 \\
\hline IGKB-2014-47 & $\begin{array}{l}\text { Palari, } \\
\text { Balaudabazar }\end{array}$ & $\begin{array}{l}\text { Branches all } \\
\text { over the stem }\end{array}$ & Low & Green & Obovate & Low & Dark Green & 7.87 \\
\hline IGKB-2014-48 & $\begin{array}{l}\text { Pandaripani, } \\
\text { Raigarh }\end{array}$ & $\begin{array}{l}\text { Branches all } \\
\text { over the stem }\end{array}$ & Low & Green & Obovate & Low & $\begin{array}{l}\text { Drak green with } \\
\text { margin pigmented }\end{array}$ & 7.13 \\
\hline IGKB-2014-49 & Raigarh & Only at base & Low & Green & $\begin{array}{l}\text { Lanceo- } \\
\text { late }\end{array}$ & Low & $\begin{array}{l}\text { Drak green with } \\
\text { margin pigmented }\end{array}$ & 6.37 \\
\hline IGKB-2014-50 & Gaitra. Tilda & $\begin{array}{l}\text { Branches all } \\
\text { over the stem }\end{array}$ & Low & Green & $\begin{array}{l}\text { Lanceo- } \\
\text { late }\end{array}$ & Low & Drak green & 11.50 \\
\hline IGKB-2014-51 & $\begin{array}{l}\text { Plari, Balaud- } \\
\text { abazar }\end{array}$ & $\begin{array}{l}\text { Branches all } \\
\text { over the stem }\end{array}$ & Medium & Green & Cuneate & Medium & $\begin{array}{l}\text { Drak green with } \\
\text { margin pigmented }\end{array}$ & 15.50 \\
\hline IGKB-2014-52 & $\begin{array}{l}\text { Pod, Abhan- } \\
\text { pur }\end{array}$ & Only at base & Medium & Green & Cuneate & Medium & Normal Green & 11.37 \\
\hline
\end{tabular}




\begin{tabular}{|c|c|c|c|c|c|c|c|c|}
\hline IGKB-2014-53 & Tulsi, Tilda & $\begin{array}{l}\text { Branches all } \\
\text { over the stem }\end{array}$ & Low & Green & Cuneate & Low & Normal Green & 15.13 \\
\hline IGKB-2014-54 & Konari, Tilda & $\begin{array}{l}\text { Branches all } \\
\text { over the stem }\end{array}$ & $\begin{array}{l}\text { Con- } \\
\text { spicious }\end{array}$ & Green & Cuneate & $\begin{array}{l}\text { Con- } \\
\text { spicious }\end{array}$ & Dark Green & 10.54 \\
\hline IGKB-2014-55 & Tila, Abhanpur & Only at base & $\begin{array}{l}\text { Con- } \\
\text { spicious }\end{array}$ & Green & Cuneate & $\begin{array}{l}\text { Con- } \\
\text { spicious }\end{array}$ & Normal Green & 14.47 \\
\hline CO1 variety & Bangalore & Only at base & Medium & Green & Lanceolate & Low & Drak Green & 15.67 \\
\hline $\mathrm{CD}(p=0.05)$ & - & - & - & - & - & - & - & 0.55 \\
\hline
\end{tabular}

Table 1: Continue

\begin{tabular}{|c|c|c|c|c|c|c|c|c|}
\hline Characters & $\begin{array}{l}\text { Leaf co- } \\
\text { lour in- } \\
\text { tensity }\end{array}$ & $\begin{array}{l}\text { Prom i - } \\
\text { nence of } \\
\text { leaf veins }\end{array}$ & $\begin{array}{l}\text { Petiole } \\
\text { pigman- } \\
\text { tation }\end{array}$ & $\begin{array}{l}\text { Terminal inflores- } \\
\text { cence Shape }\end{array}$ & $\begin{array}{l}\text { Presence of } \\
\text { axillary in- } \\
\text { florescence }\end{array}$ & $\begin{array}{l}\text { Inflorescence } \\
\text { density }\end{array}$ & $\begin{array}{l}\text { Inflores- } \\
\text { c e n c e } \\
\text { colour }\end{array}$ & Seed colour \\
\hline IGKB-2014-31 & Medium & Smooth & Green & $\begin{array}{l}\text { Panicle with short } \\
\text { branches }\end{array}$ & Present & Intermediate & Green & Jet black \\
\hline IGKB-2014-32 & Medium & Smooth & Green & $\begin{array}{l}\text { Panicle with long } \\
\text { branches }\end{array}$ & Present & Low & Green & Jet Black \\
\hline IGKB-2014-33 & Medium & Smooth & Green & $\begin{array}{l}\text { Panicle with short } \\
\text { branches }\end{array}$ & Present & Low & Green & Jet Black \\
\hline IGKB-2014-34 & Light & Smooth & Green & $\begin{array}{l}\text { Panicle with short } \\
\text { branches }\end{array}$ & Present & Low & Green & Black \\
\hline IGKB-2014-35 & Dark & Smooth & Green & $\begin{array}{l}\text { Panicle with short } \\
\text { branches }\end{array}$ & Present & Low & Green & Black \\
\hline IGKB-2014-36 & Dark & Smooth & Green & $\begin{array}{l}\text { Panicle with short } \\
\text { branches }\end{array}$ & Present & Low & Green & Black \\
\hline IGKB-2014-37 & Light & Smooth & Green & $\begin{array}{l}\text { Panicle with short } \\
\text { branches }\end{array}$ & Present & Low & Green & Black \\
\hline IGKB-2014-38 & Dark & Smooth & Green & $\begin{array}{l}\text { Panicle with short } \\
\text { branches }\end{array}$ & Present & Low & Green & Black \\
\hline IGKB-2014-39 & Light & Smooth & Green & Spike & Present & Low & Green & Black \\
\hline IGKB-2014-40 & Light & Smooth & Green & $\begin{array}{l}\text { Panicle with short } \\
\text { branches }\end{array}$ & Present & Low & Green & Black \\
\hline IGKB-2014-41 & Light & Smooth & Green & Spike & Present & Low & Green & Black \\
\hline IGKB-2014-42 & Light & Smooth & Green & Spike & Present & Low & Green & Reddish Black \\
\hline IGKB-2014-43 & Dark & Smooth & Green & Short branches & Present & Intermediate & Green & Reddish Black \\
\hline IGKB-2014-44 & Light & Smooth & Green & Short branches & Present & Intermediate & Green & Reddish Black \\
\hline IGKB-2014-45 & Medium & Smooth & Green & Spike & Present & Dense & Green & Reddish Black \\
\hline IGKB-2014-46 & Dark & Smooth & Green & Long branches & Present & Dense & Green & Reddish Black \\
\hline IGKB-2014-47 & Dark & Smooth & Green & Short branches & Present & Low & Green & Reddish Black \\
\hline IGKB-2014-48 & Medium & Smooth & Green & $\begin{array}{l}\text { Panicle with long } \\
\text { branches }\end{array}$ & Present & Low & Green & Reddish Black \\
\hline IGKB-2014-49 & Dark & Smooth & Green & $\begin{array}{l}\text { Panicle with long } \\
\text { branches }\end{array}$ & Present & Low & Green & Reddish Black \\
\hline IGKB-2014-50 & Light & Smooth & Green & $\begin{array}{l}\text { Panicle with long } \\
\text { branches }\end{array}$ & Present & Low & Green & Brown Black \\
\hline IGKB-2014-51 & Light & Smooth & Green & $\begin{array}{l}\text { Panicle with short } \\
\text { branches }\end{array}$ & Present & Low & Green & Reddish Black \\
\hline
\end{tabular}




\begin{tabular}{|c|c|c|c|c|c|c|c|c|}
\hline Characters & $\begin{array}{l}\text { Leaf co- } \\
\text { lour in- } \\
\text { tensity }\end{array}$ & $\begin{array}{l}\text { Prom i - } \\
\text { nence of } \\
\text { leaf veins }\end{array}$ & $\begin{array}{l}\text { Petiole } \\
\text { pigman- } \\
\text { tation }\end{array}$ & $\begin{array}{l}\text { Terminal inflores- } \\
\text { cence Shape }\end{array}$ & $\begin{array}{l}\text { Presence of } \\
\text { axillary in- } \\
\text { florescence }\end{array}$ & $\begin{array}{l}\text { Inflorescence } \\
\text { density }\end{array}$ & $\begin{array}{l}\text { Inflores- } \\
\text { c e n c e } \\
\text { colour }\end{array}$ & Seed colour \\
\hline IGKB-2014-52 & Medium & Smooth & Green & $\begin{array}{l}\text { Panicle with short } \\
\text { branches }\end{array}$ & Present & Dense & Green & Brown Black \\
\hline IGKB-2014-53 & Light & Smooth & Green & Spike & Present & Low & Green & Brown Black \\
\hline IGKB-2014-54 & Light & Smooth & Green & Spike & Present & Low & Green & Brown Black \\
\hline IGKB-2014-55 & Light & Smooth & Green & Spike & Present & Low & Green & Brown Black \\
\hline CO1 variety & Dark & Smooth & Green & $\begin{array}{l}\text { Panicle with long } \\
\text { branches }\end{array}$ & Present & Intermediate & Green & Black \\
\hline
\end{tabular}
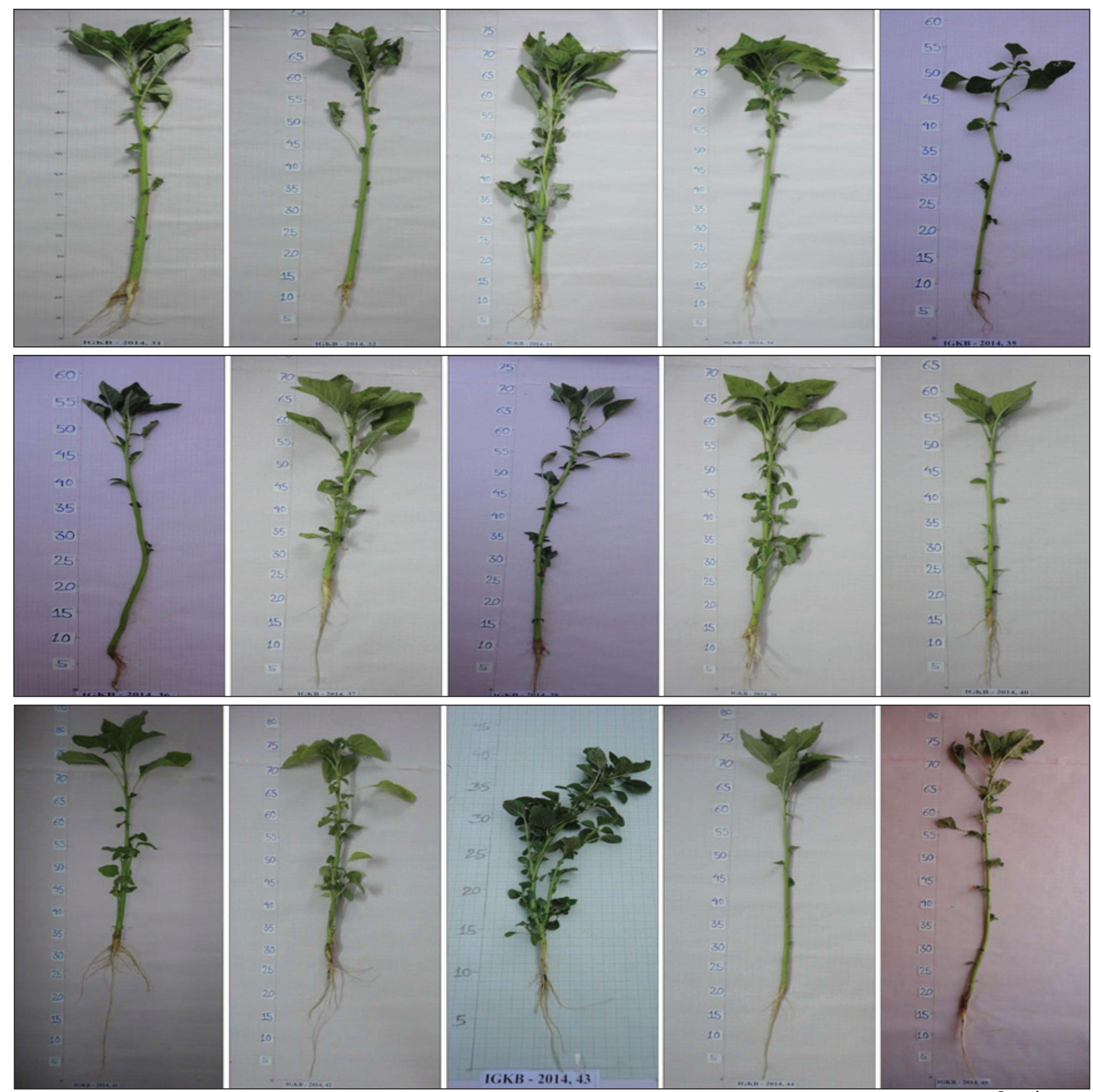

Continue... 


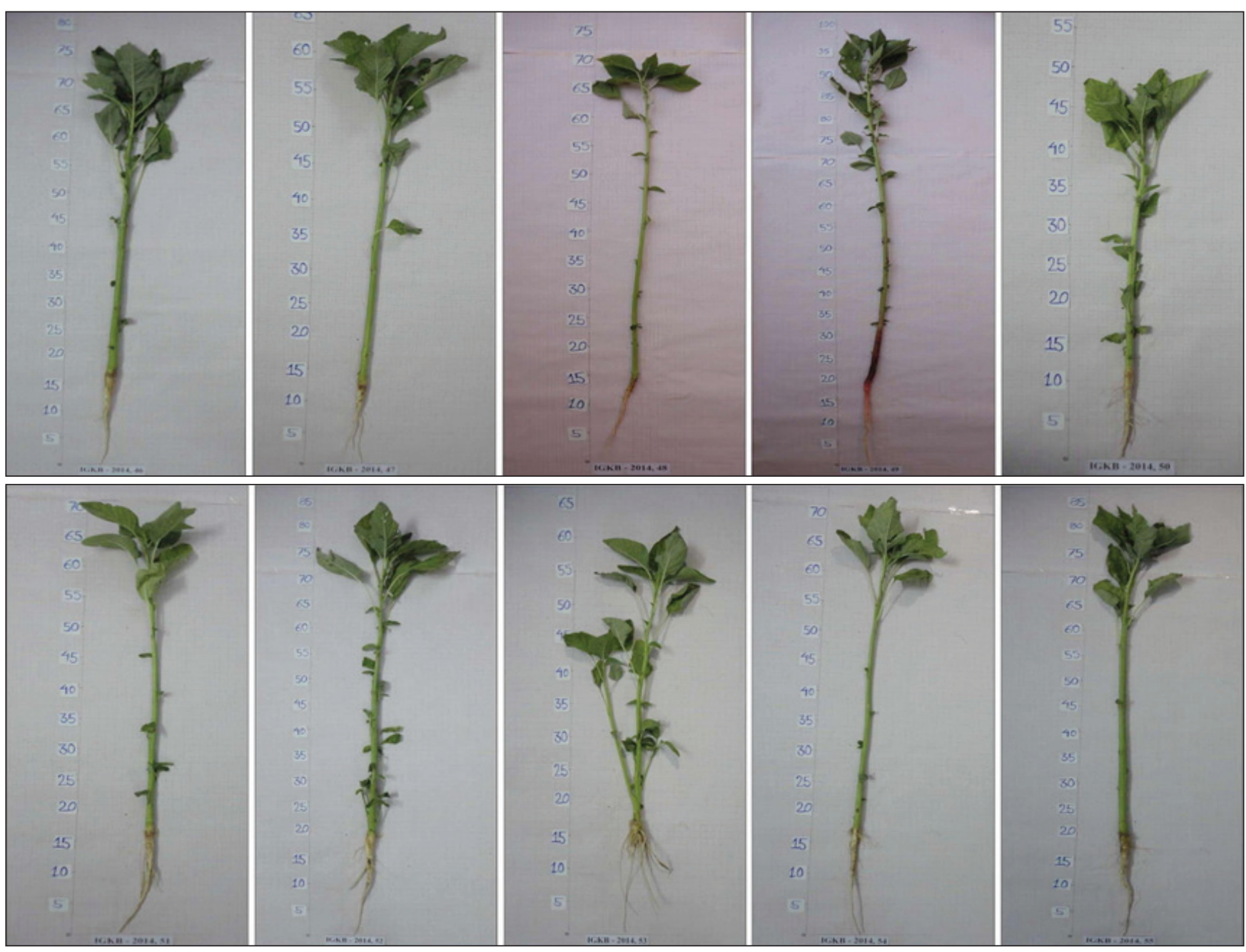

Figure 1: Collection and morphological variability in Amaranthus dubius Mart. (khedha)

IGKB-2014-53, IGKB-2014-54 and IGKB-2014-55) while, eight genotypes had dark green colour (IGKB-2014-35, IGKB2014-36, IGKB-2014-38, IGKB-2014-43, IGKB-2014-46, IGKB2014-47, IGKB-2014-49 and variety CO-1), six genotypes had medium green colour (IGKB-2014-31, IGKB-2014-32, IGKB2014-33, IGKB-2014-45, IGKB-2014-48 and IGKB-2014-52) respectively.

Total 25 genotypes and variety CO- 1 of khedha were evaluated for the prominence of leaf veins were categorized into various distinct group viz., twenty-five genotype and variety CO-1 showed smooth vein.

The 25 genotypes and variety $\mathrm{CO}-1$ of khedha were evaluated for the petiole pigmentation were categorized into various distinct group viz., twenty-five genotype and variety CO-1 had showed green petiole pigmentation.

The terminal inflorescence shape were categorized into three group viz., six genotype had panicle with long branches (IGKB-2014-32, IGKB-2014-48, IGKB-2014-49, IGKB-2014-50, IGKB-2014-46 and variety CO-1) while, thirteen genotypes had panicle with short branches (IGKB-2014-31, IGKB-2014-33,
IGKB-2014-34, IGKB-2014-35, IGKB-2014-36, IGKB-2014-37, IGKB-2014-38, IGKB-2014-40, IGKB-2014-43, IGKB-201444, IGKB-2014-47, IGKB-2014-51 and IGKB-2014-52), seven genotypes had spike (IGKB-2014-39, IGKB-2014-41, IGKB2014-42, IGKB-2014-45, IGKB-2014-53, IGKB-2014-54 and IGKB-2014-55) respectively.

The 25 genotypes and variety $\mathrm{CO}-1$ of khedha were evaluated for the presence of axillary inflorescence was present in all genotypes.

The inflorescence density were categorized into three group viz., ninteen genotype had low (IGKB-2014-32, IGKB-2014-33, IGKB-2014-34, IGKB-2014-35, IGKB-2014-36, IGKB-2014-37, IGKB-2014-38, IGKB-2014-39, IGKB-2014-40, IGKB-2014-41, IGKB-2014-42, IGKB-2014-47, IGKB-2014-48, IGKB-2014-49, IGKB-2014-50, IGKB-2014-51, IGKB-2014-53, IGKB-2014-54 and IGKB-2014-55), while, four genotypes had intermediate (IGKB-2014-31, IGKB-2014-43, IGKB-2014-44 and variety CO1 ), three genotype had dense (IGKB-2014-45, IGKB-2014-46 and IGKB-2014-52), respectively.

The 25 genotypes and variety $\mathrm{CO}-1$ of khedha were evaluated 
for the inflorescence colour were categorized into various distinct group viz., twenty-five genotype and variety CO-1 showed green colour respectively.

The seed colour were categorized into four group viz., three genotype had jet black (IGKB-2014-31, IGKB-2014-32 and IGKB-2014-33), while, nine genotypes had black colour (IGKB-2014-34, IGKB-2014-35, IGKB-2014-36, IGKB-2014-37, IGKB-2014-38, IGKB-2014-39, IGKB-2014-40, IGKB-201441 and variety $C O-1)$, nine genotypes had reddish black (IGKB-2014-42, IGKB-2014-43, IGKB-2014-44, IGKB-2014-45, IGKB-2014-46, IGKB-2014-47, IGKB-2014-48, IGKB-2014-49 and IGKB-2014-51) while, five genotypes had brown black (IGKB-2014-50, IGKB-2014-52, IGKB-2014-53, IGKB-2014-54 and IGKB-2014-55) respectively.

The obtained data expressed maximum fibre content in variety of CO-1 (15.67\%) followed by genotype IGKB-2014-51 (15.50 $\%)$ and IGKB-2014-53 (15.13 \%) was observed. Variety CO-1 and genotype IGKB-2014-51 are statically at par with each other.

\section{Conclusion}

This experiment was undertaken to assess the variability that exist on the selected amaranth germplasm collection at NPGRL in terms of its morphological features. A total of 25 accessions and check variety $\mathrm{CO}-1$ were used in the study. Amaranthus could play an important role in rural areas because its cultivation allows good yields of high quality grain and leaves to be integrated in daily poor diet. They have short crop duration (30-40 days), making them adapted to fit into peri-urban, homestead or cereal-based production systems.

\section{References}

Anonymous, 2013-14. Area, Production and Productivity. Directorate of Horticulture, Government of Chhattisgarh, Raipur (C.G.). (Chhattisgarh State Horticulture Department).

Anonymous, 2015-16a. Area, Production and Productivity. Directorate of Horticulture, Government of Chhattisgarh, Raipur (C.G.). (Chhattisgarh State Horticulture Department).

Anonymous, 2014-15. Indian Horticulture Database, National Horticulture Board, Gurgaon.

Chauhan, D., Shrivastava, A.K., Patra, S., 2014. Diversity of leafy vegetablesused by tribal peoples of Chhattisgarh India. International Journal of Current Microbiology and Applied Science 3(4), 611-622.

Singh, H.B., Arora, R.K., 1978. Wild edible plants of India $1^{\text {st }}$ ed. ICAR Publication, New Delhi, 88.

Shukla, S., Singh, S.P., 2003. Correlation and path analysis in grain amaranth (Amaranthus spp.), Indian Journal of Genetics and Plant Breeding 63(2), 163-164.

Yadav, R., Rana, J.C., Ranjan, J.K., 2014. Analysis of variability parameters formorphological and agronomic traits in grain amaranth (Amaranthus sp.) genotypes, The Bioscan, 9(4), 1661-1665. 